\title{
MATEMOS AL BICHO: LA HUERTA ESCOLAR COMO ESCENARIO EDUCATIVO PARA LA ARGUMENTACIÓN YLATOMA DE DECISIONES
}

\section{Let's Kill the Bug: the School Garden as an Educational Scenario for Argumentation and Decision Making}

\author{
Vamos matar o insecto: a horta escolar como cenário \\ educacional para argumentação e tomada de decisão
}

\section{Gerardo Juan Guerrero* Gonzalo Peñaloza Jiménez ${ }^{\star \star}$}

\section{Resumen}

El huerto escolar ha sido una estrategia ampliamente utilizada como recurso didáctico para la enseñanza de la biología y para la educación ambiental. Este artículo de investigación explora las posibilidades de dicho escenario educativo para plantear dilemas éticos y ambientales en los que se relacionan intereses y beneficios, y que implican decisiones sobre la vida y la muerte de un organismo. Para esto se llevó a cabo una investigación con estudiantes de los grados tercero ( $\mathrm{N}=21$ ) y sexto ( $\mathrm{N}=42$ ) de educación básica, quienes se involucraron en el cultivo de una huerta y en discusiones sobre dilemas en torno a la conservación o erradicación de un insecto en la huerta. A través de las justificaciones de sus decisiones, se evidencian las posibilidades que tiene este escenario para el desarrollo de las habilidades argumentativas y de toma de decisiones. Los hallazgos sugieren que los estudiantes privilegian el dominio afectivo sobre el cognitivo en sus justificaciones, es decir, que los estudiantes no incluyen frecuentemente conceptos biológicos en las razones que son parte de sus argumentos.

Palabras clave: argumentación; educación ambiental; enseñanza; didáctica; educación básica

* Centro de Investigaciones y de Estudios Avanzados del IPN (Cinvestav), Unidad de Monterrey. Correo electrónico: juan.guerreroh@cinvestav.mx

** Centro de Investigaciones y de Estudios Avanzados del IPN (Cinvestav), Unidad de Monterrey. Correo electrónico: g.pjimenez@cinvestav.cm 


\section{Abstract}

The school garden has been a widely used strategy as a teaching resource for the teaching of biology and for environmental education. This research article explores the possibilities of this educational scenario to raise ethical and environmental dilemmas in which interests and benefits are related, and that involve decisions about the life and death of an organism. For this, an investigation was carried out with students in the third $(\mathrm{N}=21)$ and sixth $(\mathrm{N}=42)$ grades of basic education, who were involved in the cultivation of an orchard and in discussions about dilemmas around conservation or eradication of an insect in the garden. Through the justifications of their decisions, the possibilities of this scenario for the development of argumentative and decision-making skills are evidenced. The findings suggest that students privilege the emotional domain over the cognitive in their justifications, that is, that students do not frequently include biological concepts in the reasons that are part of their arguments.

Keywords: argumentation; environmental education; teaching; didactics; basic education

\section{Resumo}

A horta escolar tem sido uma estratégia amplamente utilizada como recurso didático para o ensino de biologia e educação ambiental. Este artigo de pesquisa explora as possibilidades desse cenário educacional de suscitar dilemas éticos e ambientais nos quais interesses e benefícios estão relacionados, e que envolvem decisões sobre a vida e a morte de um organismo. Para isso, foi realizada uma investigação com os alunos do terceiro $(N=21)$ e sexto $(N=42)$ de ensino fundamental, envolvidos no cultivo de uma horta e nas discussões sobre dilemas em torno da conservação ou erradicação de um inseto na horta. Por meio das justificativas de suas decisões, são evidenciadas as possibilidades desse cenário para o desenvolvimento de habilidades argumentativas e de tomada de decisão. As descobertas sugerem que os alunos privilegiam o domínio afetivo sobre o cognitivo em suas justificativas, ou seja, que os estudantes não incluem frequentemente conceitos biológicos nas razões que fazem parte de seus argumentos.

Palavras-chave: argumentação; educação ambiental; ensino; didáctica; educação básica 


\section{Introducción}

En los últimos siglos las actividades humanas han venido causando un impacto global y progresivo sobre casi todos los ecosistemas, con un efecto perjudicial. De no transformarse la tendencia actual, la vida en el planeta está en riesgo de cambiar drásticamente y la existencia de los seres humanos está en peligro (Stevenson, 2018). Esto ha generado un reconocimiento casi innegable y generalizado de la importancia de la educación ambiental en la promoción de valores, actitudes y acciones conscientes sobre la naturaleza (Stone y Barlow, 2005; Villanueva, Villaroel y Antón, 2018). Esto supone que, en la medida en que los sujetos reflexionen acerca del impacto de las acciones humanas sobre el entorno, estarán más inclinados a comprometerse y a exigir que el Estado ejerza un control creciente y ambientalmente responsable sobre las actividades que vayan en detrimento de la naturaleza. Es decir, la educación ambiental viene tomando cada vez mayor relevancia en la formación básica de cualquier sujeto.

Al respecto, uno de los objetivos centrales de la educación ambiental es preparar a los estudiantes para participar activamente en la sociedad, para lo cual se requiere de individuos informados con capacidad para tomar decisiones e incidir en las decisiones sociales. En este proceso es fundamental la capacidad de generary comprender argumentos. Es decir, es crucial el desarrollo de habilidades que les permitan usar el conocimiento para proponer soluciones que discriminen entre las necesidades humanas generales, los intereses de sectores particulares y el efecto que una acción puede tener en la naturaleza, entre otras.

Desde el punto de vista de la educación se supone que una persona que logre un nivel multidimensional de alfabetización científica debería estar en capacidad de establecer conexiones entre su comprensión conceptual (uso del dominio cognitivo), sus actitudes y creencias (uso del dominio afectivo) y las decisiones que toma (uso del dominio del comportamiento) (Liem, 2005). En el caso de la educación ambiental, se deben crear escenarios en los que los niños se vean compelidos a poner en juego estos dominios y desarrollen las habilidades consecuentes.

Sin embargo, no se ha explorado exhaustivamente cómo diferentes estudiantes (de diversos contextos culturales, educativos, etc.) toman decisiones, cómo las justifican y qué papel le dan a la evidencia (Evagorou, Jiménez-Aleixandre y Osborne, 2012). Por ello, en este artículo se presentan los resultados preliminares de un proyecto de investigación sobre el uso de la argumentación en torno a cuestiones ambientales que involucran la interacción ecológica hombre-hortaliza-larva. Particularmente, se muestra cómo la huerta escolar es un escena- rio que genera dilemas sobre la preservación de la vida, las necesidades humanas y su impacto en la naturaleza, las cuales pueden ponerse en función del desarrollo de habilidades argumentativas. Al respecto se estudiaron las justificaciones que dan los estudiantes a las soluciones planteadas por ellos ante el dilema de matar o no a una larva de coleóptero.

\section{Conocimiento, decisiones y acción}

En el marco de la perspectiva constructivista de la educación, se considera que los escenarios educativos deben estar diseñados de manera que propongan situaciones que permitan que los sujetos desarrollen comprensiones sobre cierta cuestión y reflexionen acerca de sus criterios para actuar de tal o cual manera (Sousa, 2001). Es decir, en la escuela deben crearse las condiciones necesarias para que los estudiantes puedan expresar sus ideas e intercambiar opiniones para desarrollar procesos de pensamiento crítico y argumentativo, involucrando conocimientos disciplinares en ello.

Como se indicó antes, en este proceso los estudiantes pueden desarrollar la capacidad de establecer conexiones entre su comprensión conceptual (dominio cognitivo), sus actitudes y creencias (dominio afectivo) y las decisiones que toman (dominio del comportamiento) (Liem, 2005). Con frecuencia, cuando se abordan cuestiones ambientales se apela al dominio afectivo, dejando de lado el cognitivo. No obstante, los problemas ambientales tienen un carácter científico porque, además de los juicios morales y los criterios estéticos que están involucrados en ellos, tienen un importante componente de conocimientos específicos que son claves para la toma de decisiones (Sadler, 2009).

Sin embargo, en muchas de las decisiones ambientales que se toman, las opiniones y acciones no se fundamentan en evidencias de los efectos e impactos que determinada acción puede tener en la naturaleza sino, sobre todo, en valoraciones subjetivas. Si bien esto no es en sí mismo negativo, sí reviste un riesgo cuando estas razones se esgrimen para la toma de decisiones sociales y deben contraponerse frente a argumentos que apelan a "la razón", dando la apariencia de que las acciones ecológicamente responsables son solo "buenas intenciones" que no son viables para suplir las necesidades de la sociedad. En estos casos, se evidencia la importancia de que las justificaciones involucren el dominio cognitivo y formen parte de las decisiones informadas de los sujetos (Abd-El-Khalick, 2003).

Por tanto, la educación ambiental debería contribuir a formar individuos capaces de identificar y enfrentar dilemas 
ambientales. Para esto, los sujetos deben comprender los conceptos relevantes, reconocer las conexiones personales y sociales que involucran fenómenos ecológicos y reconocer las decisiones y acciones consecuentes con la resolución del dilema (Balgopal y Wallace, 2009). En otras palabras, un sujeto ambientalmente consciente es aquel que está en capacidad de comprender los conceptos científicos más importantes y tomar decisiones sobre la base de tal comprensión (Van Weelie, 2002). Desde luego, esto lo hará tomando en cuenta su sistema de valores y su visión del mundo (Grace y Ratcliffe, 2002). Este tipo de decisiones se definen como elecciones razonadas, que se soportan en criterios que se construyen mediante la discusión y la reflexión (Kortland, 1997).

En relación con las justificaciones, cabe señalar que ellas forman parte de la argumentación, entendida como un proceso de pensamiento y de expresión que, de manera retórica y lógica, se usa para defender, debatir ideas y fundamentar las opiniones con razones (Wholrapp, 2014). Este proceso, además de sus contribuciones a la formación política de los individuos, es muy relevante en la práctica científica, en la comunicación de la ciencia y en la comprensión de las teorías, los modelos, productos y aportes que ella hace (Conrado, Nunez-Neto y El-Hani, 2015).

En el marco de la teoría de Toulmin (1958), los argumentos tienen un patrón en el que hay básicamente cinco elementos: datos, afirmación, justificación, respaldo y refutación. Los datos deben dar paso a una afirmación que es justificada y cuenta con un soporte (respaldo). En el marco de la educación científica se espera que los estudiantes sean capaces de formular afirmaciones a partir de los datos, que las justificaciones soporten esa conexión entre datos y que, a su vez, se basen en supuestos básicos (soportes). No obstante, como ya se refirió, los estudiantes tienden a basar sus justificaciones en valores más que en conocimientos.

Particularmente, los estudios sobre las justificaciones que expresan los estudiantes muestran que ellos tienen dificultades para usar evidencias que respalden sus decisiones y buena parte de sus argumentos se soportan en opiniones muy subjetivas que, en el marco del proceso de argumentación, podrían no ser aceptadas por los otros sujetos involucrados en el diálogo. De hecho, se ha señalado que las opiniones de los estudiantes se relacionan estrechamente con sus valores (Evagorou, 2011; Evagorou, Jiménez-Aleixandre y Osborne, 2012; Jiménez-Aleixandre y Pereiro-Munoz, 2002), pero en un proceso dialógico los valores son un aspecto que no necesariamente es compartido por las partes, por lo tanto, si los argumentos se basan exclusivamente en ellos la contraparte podría desestimarlos.
Los dilemas pueden definirse como situaciones en las que los estudiantes deben tomar decisiones sobre acciones que afectan directamente a organismos vivos y que son cruciales para cumplir con un objetivo, en este caso, productivo. Por ejemplo, los estudiantes se ven en la necesidad de decidir si deben o no sacrificar una larva que amenaza el cultivo que están haciendo en la huerta. Antes de tomar la decisión, se les invita a conocer la larva y el efecto que su crecimiento podría tener sobre el cultivo, de modo que tengan más información al momento de verse en la necesidad de decidir.

\section{La educación ambiental en la escuela primaria}

Actualmente existe un consenso generalizado sobre la necesidad de promover actitudes y comportamientos ambientales en la población, de manera que se tomen - o se presione para que se tomen-medidas tendientes a disminuir el efecto de las actividades humanas en la naturaleza, o al menos que no perjudiquen al ambiente (López, Álvarez, González y García, 2014). Este interés ha generado investigaciones y discusiones teóricas sobre la importancia, viabilidad y la forma de desarrollar la educación ambiental en los primeros años de escolaridad (Collado, Evans, Corraliza y Sorrel, 2015). Particularmente, es notoria la necesidad de conocer cómo piensan y qué sienten los niños sobre las cuestiones ambientales y de qué manera la razón y el afecto se convierten en acciones.

Los estudios sobre las preocupaciones ambientales de las personas no son concluyentes acerca de la relación que existe entre sus actitudes, sus comportamientos y su edad. Algunos han sugerido que, conforme la edad aumenta, las actitudes favorables hacia el ambiente decrecen y que, entre los preadolescentes (9 a 13 años) se encuentran valores, creencias y comportamientos más positivos hacia la naturaleza (Pol y Castrechini, 2013). A pesar de las diferencias sobre las relaciones entre la edad y las preocupaciones por el medioambiente, se sabe que la formación inicial que en esta materia tengan los estudiantes dará forma a buena parte de sus comportamientos como adultos. Por tanto, la educación ambiental en los primeros años resulta clave.

Pese a la importancia de estas cuestiones éticas y ambientales, son pocos los estudios sobre ellas (Koch, Barkmann, Sundawati y Bögeholz, 2013; Menzel y Bogeholz, 2009). Los temas relacionados con la educación ambiental y el desarrollo sustentable implican conocimientos y consideraciones interdisciplinarias, que involucran aspectos éticos y dilemas que implican riesgos y necesidades. Por ejemplo, la conservación de la diversidad se ha convertido 
en un asunto frente al que hay un consenso generalizado. Sin embargo, cuando la conservación de la naturaleza se contrasta con la necesidad de suplir las necesidades humanas, las alternativas parecen no ser tan claras ni tan enfáticas. Esto simplemente para ilustrar que la educación científica tiene el reto de formar sujetos que puedan poner el conocimiento biológico en diálogo con el de otras disciplinas y hacer esto, en la medida de lo posible, desde la escuela primaria.

\section{La huerta escolar}

Como ya se señaló, este artículo explora las posibilidades de la huerta escolar como escenario en el que se suscitan dilemas que tienen un alcance amplio y que pueden extrapolarse a situaciones reales y globales en las que la sociedad toma decisiones sobre la vida. Se explora cómo esta estrategia puede promover la comprensión de la relación entre sociedad y naturaleza. Para esto se analizó el uso de conocimientos ecológicos relevantes para la toma de decisiones y la evaluación de argumentos e información para la toma de decisiones.

El estudio se enfocó en los argumentos en general y no solo en aquellos que involucran conocimientos científicos relevantes. De hecho, Jiménez-Aleixandre (2014) estableció que ante dilemas socioambientales los estudiantes tienden no solo a considerar argumentos de tipo conceptual, sino a involucrar sus valores. Esto, lejos de ser una desventaja, es una posibilidad para la construcción de escenarios educativos que formen a los futuros sujetos que van a decidir e influir en asuntos ambientales.

La huerta se constituye en un escenario en que los estudiantes no se limitan a ser consumidores de conocimiento, sino en el que pueden producirlo. Se sabe que los escenarios más eficaces, desde el punto de vista formativo, son aquellos en los cuales las decisiones de los participantes abren o cierran posibilidades. Las decisiones para tomar se presentan entonces como asunto de debate en torno al que se expresan diversas posibilidades. Así, estos escenarios demandan evidencias, criterios de justificación y evaluaciones (King y Ritchie, 2012).

En este sentido, la huerta escolar se convierte en un problema auténtico que, si bien no es estrictamente "real", permite motivar e interesar a los estudiantes, ofreciéndoles la posibilidad de discusión (Jiménez-Aleixandre, 2014). La huerta abordada en la perspectiva ecológica y ambiental que se propone implica, en efecto, que los estudiantes se vean en la necesidad de discutir colectivamente para tomar decisiones ante un problema auténtico que involucra seres vivos y el futuro de su proyecto colectivo.

\section{Metodología}

Esta investigación plantea un dilema ambiental haciendo referencia a la relación ecológica humano-lechuga-larva que se presenta en una huerta. Se adelantó siguiendo un enfoque cualitativo y se realizó en el marco de un curso de enseñanza de la biología para tercer y sexto grado de educación básica en una escuela privada ubicada en Monterrey, Nuevo León, México. Participaron 21 niños de tercer grado y 42 de sexto. La investigación se realizó durante 4 semanas, periodo en el que los estudiantes estuvieron involucrados en la preparación de suelo y cultivo del huerto escolar. Las actividades de los dos grupos fueron orientadas por el mismo docente, quien es el primer autor de este artículo. En la tabla 1 se resume el proceso que se llevó a cabo con los estudiantes.

Tabla 1. Etapas del proceso de investigación

\begin{tabular}{|c|c|}
\hline Sesiones & Descripción \\
\hline $\begin{array}{l}\text { Primera sesión: } \\
\text { Preparación } \\
\text { del suelo }\end{array}$ & $\begin{array}{l}\text { Los alumnos reconocieron tres tipos de suelo: } \\
\text { arena, arcilla y limo, así como la importancia de } \\
\text { preparar el suelo para el desarrollo de las raíces } \\
\text { de las plantas y la retención de agua. } \\
\text { Durante la actividad de mezcla los estudiantes } \\
\text { tuvieron la oportunidad de encontrarse con } \\
\text { algunas larvas de coleóptero y discutieron qué } \\
\text { hacer con ellas. } \\
\text { El maestro sugirió removerlas del lugar, incluso } \\
\text { sacrificarlas. Se generó una discusión in situ. }\end{array}$ \\
\hline $\begin{array}{l}\text { Segunda } \\
\text { sesión: } \\
\text { Planteamiento } \\
\text { de dilemas }\end{array}$ & $\begin{array}{l}\text { Cada alumno recibió un instrumento en el que } \\
\text { se le pidió que tomaran una decisión sobre las } \\
\text { larvas de coleóptero que se encontraron en la } \\
\text { huerta. }\end{array}$ \\
\hline $\begin{array}{l}\text { Tercera sesión: } \\
\text { Siembra en } \\
\text { almácigos } \\
\text { y siembra } \\
\text { directa }\end{array}$ & $\begin{array}{l}\text { Los alumnos preparan charolas de siembra con } \\
\text { turba, colocando un par de semillas por pozo. } \\
\text { Los alumnos siembran en zigzag un par de } \\
\text { semillas con ayuda de un cartón que les permita } \\
\text { ubicar los espacios de siembra. }\end{array}$ \\
\hline
\end{tabular}

El estudio se planteó dos preguntas fundamentales:

- ¿Qué soluciones y justificaciones plantean los estudiantes ante el dilema de sacrificar o no unas larvas en el marco de cultivar la huerta escolar?

- ¿Qué papel tienen los conocimientos sobre ecología en las soluciones y las justificaciones de los estudiantes?

Estas preguntas se enmarcan en el interés por establecer si la huerta escolar puede ser un escenario educativo para generar procesos de argumentación y de toma de decisiones entre los estudiantes. 
Para abordar el problema, se llevó a cabo un estudio cualitativo exploratorio, que se realizó en dos fases: en la primera, se diseñaron los cuestionarios que se iban a aplicar; en la segunda, se aplicaron los instrumentos y se realizó la observación. En los cuestionarios se planteó el dilema de sacrificar o no unas larvas que amenazaban con devorar el cultivo de la huerta. Además de las respuestas escritas con respecto a los dilemas, se registraron las discusiones que se suscitaron a raíz de ellos.

Para la interpretación de la información recabada se llevó a cabo un análisis del discurso orientado a reconocer los procesos de construcción y justificación de las decisiones.
Para esto se adaptó y se usó el procedimiento propuesto por Jiménez-Aleixandre y Pereiro-Munoz (2002) y Evagorou et ál. (2012) en el que se propusieron categorías para las soluciones y las justificaciones.

\section{Resultados y discusión}

Mediante la revisión de los textos de los estudiantes y su posterior interpretación se definieron algunas categorías. En la tabla 2 se presentan las decisiones planteadas por los estudiantes de tercery sexto grado. Como se evidencia en las citas que se presentan como ejemplo, las decisiones sobre las larvas fueron cuatro: reubicarlas, no moverlas, investigarlas y erradicarlas.

Tabla 2. Tipo de soluciones de los estudiantes

\begin{tabular}{|c|c|}
\hline Solución & Cita \\
\hline Reubicación & $\begin{array}{l}\text { "Que no las asusten, ni tampoco molestarlas, sino hacer lo correcto y no maltratarlas o si no les pueden hacer daño } \\
\text { y también tener una buena distancia y tratarlas como seres vivos". (Estudiante de tercer grado, n. } 5 \text { ). } \\
\text { "Yo recomendaría que muevan las larvas de escarabajo porque cuando crezcan se van a comer la vegetación. Yo } \\
\text { aconsejaría que muevan a los escarabajos a un lugar más saludable para ellos". (Estudiante sexto grado, n. }{ }^{\circ} \text { 46) }\end{array}$ \\
\hline No moverlas & $\begin{array}{l}\text { "Dejarlo en paz porque se pueden asustar. Aparte también son seres vivos. Y la huerta es su casa." (Estudiante tercer } \\
\text { grado, n. }{ }^{\circ} \text { 14). } \\
\text { "Yo recomendaría que dejen a las larvas en el suelo porque ellos les dan nutrientes a las plantas. Pero también hay } \\
\text { cosas negativas como que las larvas de escarabajo se comen varios frutos, como la lechuga y el tomate y eso no es } \\
\text { bueno." (Estudiante sexto grado, n. }{ }^{\circ} \text { 26). }\end{array}$ \\
\hline Investigarlas & $\begin{array}{l}\text { "Yo pienso que lo positivo es que son inofensivos y lo negativo es que te arruinan el huerto, que los estudien." } \\
\text { (Estudiante tercer grado, n. } 21 \text { ). } \\
\text { "Les diría que se puede construir una granja de esas larvas de escarabajos y construir un ecosistema en el jardín } \\
\text { y tendrán más tiempo de investigar sobre ellos. Yo diría eso para que investiguen si puede que hay otro tipo de } \\
\text { criatura u otro tipo de larva y si quieren construir su granja pueden saber con qué alimentarlos, cómo cuidarlos, etc. } \\
\text { (Estudiante sexto grado, n. } 49 \text { ). }\end{array}$ \\
\hline Erradicarlas & $\begin{array}{l}\text { "Yo recomendaría que las maten porque no les sirve y lo más importante es que son plaga y se van a comer todas } \\
\text { las plantas." (Estudiante sexto grado, n. } 50 \text { ). } \\
\text { "Yo les recomendaría que quiten a las gallinas ciegas (nombre coloquial que reciben en México estas larvas) porque } \\
\text { se comen las plantas". (Estudiante sexto grado, n. }{ }^{\circ} \text { 2) }\end{array}$ \\
\hline
\end{tabular}

Cabe resaltar que ningún estudiante de tercer grado planteó la posibilidad de matar a las larvas (tabla 2) a pesar de que, como se evidencia en la tabla 3, ellos reconocían el riesgo que su proliferación suponía para el cultivo. Esto indica que los estudiantes no toman en cuenta la evi- dencia del riesgo que para las plantas supone la presencia de larvas e ignoran esta justificación, dándole mayor importancia al hecho de que las larvas "son seres vivos". De hecho, en la tabla 2, se evidencia que estas dos justificaciones tuvieron la misma frecuencia. 
Tabla 3. Frecuencia del tipo de solución

\begin{tabular}{|l|c|c|c|c|}
\hline \multicolumn{1}{|c|}{ Solución } & $\begin{array}{c}\text { Tercer } \\
\text { grado } \\
(\mathbf{N = 2 1 )}\end{array}$ & $\%$ & $\begin{array}{c}\text { Sexto } \\
\text { grado } \\
(\mathbf{N}=\mathbf{4 2})\end{array}$ & $\%$ \\
\hline Reubicación & 6 & 29 & 19 & 45 \\
\hline No moverlas & 10 & 48 & 11 & 26 \\
\hline Investigarlas & 2 & 9 & 5 & 12 \\
\hline Erradicarlas & 0 & 0 & 4 & 10 \\
\hline Sin solución & 3 & 14 & 3 & 7 \\
\hline
\end{tabular}

En el caso de los estudiantes de sexto grado, se conserva el valor sobre la larva como ser vivo, siendo la justificación con mayor incidencia (tabla 3); sin embargo, en esta ocasión también podemos apreciar que ahora le brindan los mismos atributos a la planta como ser vivo. Esto puede tener una relación con el hecho de que el $45 \%$ de los estudiantes de sexto grado decide reubicarlas, mientras que en tercer grado solo el $29 \%$ considera esta opción. En este mismo grupo, también aparece la sugerencia de erradicarlas por el daño que pueden representar para el desarrollo de la huerta, otorgando mayor valor a la planta. La segunda justificación de mayor frecuencia esta relacionada con la investigación de la naturaleza propia de la larva (tabla 3), mientras que los chicos de tercer grado a pesar de proponer a la investigación como recomendación, no la justifican. En la tabla 3 podemos apreciar que el número de justificaciones se diversifica en los alumnos de sexto grado.

Tabla 4. Frecuencia de las justificaciones de los estudiantes

\begin{tabular}{|l|c|c|}
\hline \multicolumn{1}{|c|}{ Justificación } & $\begin{array}{c}\text { Tercer grado } \\
\text { (N = 21) }\end{array}$ & $\begin{array}{c}\text { Sexto grado } \\
\text { (N = 42) }\end{array}$ \\
\hline Ocasiona daños al huerto. & 9 & 10 \\
\hline $\begin{array}{l}\text { Es un ser vivo. } \\
\text { No es perjudicial para los } \\
\text { humanos. }\end{array}$ & 9 & 19 \\
\hline $\begin{array}{l}\text { Es perjudicial para los } \\
\text { humanos. }\end{array}$ & 0 & 2 \\
\hline $\begin{array}{l}\text { Se convertirá en una } \\
\text { mariposa. }\end{array}$ & 1 & 4 \\
\hline \begin{tabular}{l} 
Investigar su naturaleza. \\
\hline Podrían favorecer la huerta.
\end{tabular} & 0 & 1 \\
\hline No sirve para nada. & 0 & 11 \\
\hline Ninguna justificación & 9 & 3 \\
\hline
\end{tabular}

Este hallazgo puede interpretarse a la luz de dos elementos. En primer lugar, algunos estudios han demostrado que en los primeros años los niños tienen dificultades para otorgarles a las plantas el mismo estatus de ser vivo que les asignan a los animales (Leddon, Waxman y Medin, 2009). De acuerdo con estos autores esta diferenciación no es absoluta y está mediada por los términos que se usen. De modo que los niños sí pueden considerar a las plantas como una "cosa o entidad viva" (living thing), pero tienen problemas en concebirlas como un "ser vivo" (alive), esto último más asociado con el hecho de tener movimiento. En el caso aquí tratado esto se expresa en que, por ejemplo, a las larvas se les otorgan sentimientos: "es una amiga, no enemiga y tiene sentimientos" (Estudiante n. ${ }^{\circ}$ 8 , grado $\left.3^{\circ}\right)^{)}$, algo que no hacen con las plantas.

En segundo lugar, y en estrecha relación con el primer elemento, en los niños de tercer grado parece predominar un sentido de preservación de la vida. Así, en la medida en que consideran que las plantas no están vivas, o por lo menos que no "sienten" igual que la larva, deciden preservar la vida de esta última, a costa de la vida de las plantas. Esta decisión y las justificaciones asociadas parecen mostrar que efectivamente el estatus de "ser vivo" implica juicios de valor diferenciales para estos dos grupos (animales y plantas).

Si bien esta diferenciación no pareciera tan importante, si se extrapola a cuestiones socio-científicas más generales, se evidencia que puede tener consecuencias en la toma de decisiones en dilemas en los que se contraponga la conservación de las plantas frente a la de los animales. Por ejemplo, en algunos casos se ha documentado que la sobrepoblación de ciervos afecta negativamente a los bosques naturales por lo que se permite la caza y se promueve como mecanismo para controlar la población (Relva y Sanguinetti, 2016). Es probable que, en una situación como esta, los individuos se muestren en contra de esta medida por la empatía que se genera con los animales más que con las plantas.

En la tabla 2 se evidencia que buena parte de los estudiantes de tercer grado (10) propusieron que las dejaran en la huerta y no las perturbaran: "yo les recomendaría dejarlas ahí porque son seres vivos y también cuidarlas muy bien como si fueran hermanos y no hacerles daño" (Estudiante n. ${ }^{\circ}$, grado $3 .^{\circ}$ ). Es decir, se genera mayor empatía con la larva que con las plantas, en comparación con los alumnos de sexto grado, quienes ya consideran la conservación de las hortalizas.

En una investigación llevada a cabo por Evagorou et ál. (2012) con estudiantes de entre 12 y 13 años en la que se les planteó un dilema sobre eliminar una población de una especie de ardillas que tenía en riesgo la población de otra especie endémica de ardillas sí se encontró que para la mayoría resulta reprochable matar a las ardillas invasoras. Incluso a pesar de que las evidencias sugieren que esto ocasionará la extinción de las ardillas endémicas. En comparación con nuestro estudio, los alumnos 
de entre 11 y 12 años toman decisiones con el fin de preservar la vida de ambos organismos, larva y planta. Esto podría suponerse a razón del uso que se da a las hortalizas o bien por un sentido afectivo al ser los alumnos quienes se involucran en el ciclo vital de la planta.

Entonces, se puede afirmar que las decisiones de los estudiantes de tercer grado se basan en otorgarles a las larvas un estatus distinto al de las plantas y en privilegiar la preservación de su vida, por encima de la de las plantas. Esto es acorde con otros estudios que sugieren que el sistema de creencias de los estudiantes tiene expresión y gran importancia en el momento de tomar decisiones ante dilemas como el que aquí se planteó (Collado et ál., 2015; Evagorou, 2011; Evagorou et ál., 2012; Jiménez-Aleixandre y Pereiro-Munoz, 2002).

En los alumnos de sexto grado también podemos apreciar la presencia de categorías como la contraargumentación, el refuerzo y la conclusión (Betancur, 2009). Esto se evidencia en la solución del alumno n. ${ }^{\circ} 49$ (tabla 1), quien refuerza su postura al sugerir futuras investigaciones, $y$ en lo que expresa el alumno n. ${ }^{\circ} 29$ :

Yo les recomendaría que los dejen libres, pero lejos del huerto ya que ellos son seres vivos y merecen vivir, pero también tienen que proteger el huerto para que crezca de la manera correcta. Cuando se conviertan en escarabajos podrían destruir el huerto, así que mantener la distancia entre el huerto y las larvas es una estupenda idea en mi opinión.

En este estudio exploratorio se revisó el rol que cumplen el dominio afectivo y el cognitivo en las justificaciones que plantean los estudiantes. Esto permite, de alguna manera, inferir su preponderancia en el momento de tomar decisiones, en este caso, frente a la sobrevivencia de una larva.

Probablemente, las discusiones colectivas sobre los efectos de las larvas en el cultivo pueden contribuir a que los estudiantes establezcan una ponderación diferente sobre el hecho de que la vida no se puede preservar siempre y que, inevitablemente, la proliferación de las larvas va a acabar con la vida de las plantas y no permitirá llevar a buen término el cultivo. Como se ha señalado, la huerta escolar es un punto de partida para plantear una cuestión socio-científica más general en la que, por ejemplo, puede plantearse la necesidad de producir alimentos para el consumo humano. En este caso puede ser más patente que deben tomarse decisiones sobre el control de plagas y que soluciones como "la reubicación" no son viables.

\section{Conclusiones}

Las experiencias culturales y las identidades personales tienen gran influencia en el tipo de decisiones que los sujetos toman. En este sentido, se evidencia la necesidad de reconocer otros contextos, como el de las comunidades rurales, donde los niños, por el tipo de relación que establecen con la naturaleza, pueden plantear otro tipo de soluciones y justificaciones. Por ejemplo, podrían reconocer el problema que supone una plaga para la economía familiar que depende de la cosecha de un cultivo.

Las decisiones planteadas por los estudiantes están fundadas en un sistema de valores, y si bien los datos de este estudio no son concluyentes, sí están en el mismo sentido de otros hallazgos que sugieren que los sujetos usan solamente las evidencias que refuerzan sus decisiones e ignoran las demás. Esto supone un reto para la educación en ciencias, porque implica que se deben fomentar el desarrollo de pensamiento crítico y la autorreflexión para cuestionar los supuestos personales y tomar en cuenta las evidencias que los contradicen.

\section{Referencias}

Abd-El-Khalick, F. (2003). Socioscientific Issues in Pre-college Science Classrooms. En D. Zeidler (ed.), The Role of Moral Reasoning and Discourse on Socioscientific Issues in Science Education (pp. 41-62). Dordrecht: Kluwer Academic.

Balgopal, M. y Wallace, A. (2009). Decisions and Dilemmas: Using Writing to Learn Activities to Increase Ecological Literacy, Journal of Environmental Education, 40(3), 13-26. DOI:10.3200/JOEE.40.3.13-26.

Betancur, L. (2009). El desarrollo de la competencia argumentativa. Textos. Didáctica de la Lengua y de la Literatura, 29, 22-26.

Collado, S., Evans, G., Corraliza, J. y Sorrel, M. A. (2015). The Role Played by Age on Children's Pro-ecological Behaviors: An Exploratory Analysis. J. Environ. Psychol, 44, 85-94.

Conrado, D. Nunez-Neto, N. y El-Hani, C. (2015). Argumentação sobre problemas socioambientais no ensino de biologia. Educacao em Revista, 31(1), 329-357.

Evagorou, M. (2011). Discussing a Socioscientific Issue in a Primary School Classroom: The Case of Using a Technology-Supported Environment in Formal and Nonformal Settings. En T. Sadler (ed.), Socio-scientific Issues in the Classroom (pp. 133-160). Londres: Springer. 
Evagorou, M., Jiménez-Aleixandre, M. P. y Osborne, J. (2012). 'Should We Kill the Grey Squirrels?' A Study Exploring Students' Justifications and Decision-making. International Journal of Science Education, 34(3), 401-428. DoI: 10.1080/09500693.2011.619211.

Grace, M., y Ratcliffe, M. (2002). The Science and Values Young People Draw Upon to Make Decisions About Biological Conservation Issues. International Journal of Science Education, 24, 1157-1169.

Jiménez-Aleixandre, M. P. y Pereiro-Munoz, C. (2002). Knowledge Producers or Knowledge Consumers? Argumentation and Decision Making About Environmental Management. International Journal of Science Education, 24(11), 1171-1190.

Jiménez-Aleixandre, M.P. (2014). Determinism and Underdetermination in Genetics: Implications for Students' Engagement in Argumentation and Epistemic Practices. Science \& Education, 23(2), 465-484

King, D. y Ritchie, S. (2012). Learning Science Through Real-World Contexts. En B. Fraser, K. Tobin y C. McRobbie. (eds.), Second International Handbook of Science Education (pp. 69-79). Dordrecht: Springer.

Koch, S., Barkmann, J., Sundawati, L. y Bögeholz, S. (2013). Subjective Theories of Indonesian Agronomy and Biology Teacher Students on Environmental Commons Dilemmas. International Research in Geographical and Environmental Education, 22(2), 120-138. DOI: 10.1080/10382046.2013.779123.

Kortland, K. (1997). Garbage: Dumping, Burning and Reusing/Recycling: Students' Perception of the Waste Issue. International Journal of Science Education, 19, 65-77.

Leddon, E. M., Waxman, S. R. y Medin, D. L. (2009). Unmasking "alive": Children's Appreciation of a Concept Linking all Living Things. J. Cogn. Dev., 9, 461-473.

Liem, A. (2005). Promoting Science Literacy by Engaging the Public. PLoS Biol 3(12), e427.

López, M., Álvarez, P., González, E. y García, M. (2014). Medidas del comportamiento ecológico y antecedentes: conceptualización y validación empírica de escalas. Universitas Psychologica, 14(1), 189204. https://revistas.javeriana.edu.co/index.php/ revPsycho/article/view/4796.
Menzel, S. y Bogeholz, S. (2009). The Loss of Biodiversity as a Challenge for Sustainable Development: How do Pupils in Chile and Germany Perceive Resource Dilemmas? Research in Science Education, 39(4), 429-447.

Pol, E. y Castrechini, A. (2013). ¿Disrupción en la educación para la sostenibilidad? Revista Latinoamericana de Psicología, 45(3), 335-349. DoI: 10.14349/rlp. v45i3.1477.

Relva, M. y Sanguinetti, J. (2016). Ecología, impacto y manejo del ciervo colorado (Cervus elaphus) en el noreste de la Patagonia Argentina. Mastozoología Neotropical, 23(2), 221-238.

Sadler, T. (2009). Socioscientific Issues in Science Education: Labels, Reasoning and Transfer. Cultural Studies in Science Education, 4(3), 705-711.

Sousa, D. A. (2001). How the brain learns. Thousand Oaks, USA: Sage

Stevenson, H. (2018). Global Environmental Politics Problems, Policy and Practice. United Kingdom: Cambridge University Press.

Toulmin, S. (1958). The Uses of Argument. Cambridge: Cambridge University Press.

Stone, M. K. y Barlow, Z. (2005). Ecological Literacy: Educating Our Children For a Sustainable World. San Francisco: Sierra Club Books.

Van Weelie, D. (2002). Making Biodiversity Meaningful Through Environmental Education. International Journal of Science Education, 24, 1143-1156.

Villanueva, S., Villaroel J. y Antón, A. (2018). Environmental Awareness and Its Relationship with the Concept of the Living Being: A Longitudinal Study. Sustainability, 10, 1-10. DoI: 10.3390/su10072358.

Villaroel, J. D., Antón, Á., Zuazagoitia, D. y Nuño, T. (2017). Young Children's Environmental Judgement and Its Relationship with their Understanding of the Concept of Living Things. Environ. Socio-Econ. Stud., 5, 1-10.

Wholrapp, H. (2014). The concept of argument. Dordrecht: Springer. 